\title{
Novel Ablation Strategy for Isolating the Superior Vena Cava Using Ultra High-Resolution Mapping
}

\author{
Yasuaki Tanaka, MD; Atsushi Takahashi, MD; Takamitsu Takagi, MD; Jun Nakajima, MD; \\ Katsumasa Takagi, MD; Hiroyuki Hikita, MD; Masahiko Goya, MD; Kenzo Hirao, MD
}

\begin{abstract}
Background: The aim of this study was to categorize the conduction patterns between the right atrium (RA) and the superior vena cava (SVC), and to determine the ideal procedure for SVC isolation using a novel high-resolution mapping system.
\end{abstract}

\begin{abstract}
Methods and Results: RA-SVC conduction was evaluated using the RHYTHMIA system in 113 patients (age $62.8 \pm 11.5$ years, paroxysmal: 67) with atrial fibrillation (AF) after pulmonary vein (PV) isolation. In 56 patients, a line of conduction block was found to run obliquely just above the sinus node (Block group). The remaining 57 patients did not have block (Non-block group). Non-PV foci were spontaneous or provoked with isoproterenol after electrical cardioversion of pacing-induced AF. In 43 patients with SVC foci (Block group: 22, Non-Block group: 21), SVC was isolated by radiofrequency applications delivered along the line connecting the open ends of the block line (Block group) or by conventional methods (Non-block group). The Block group required fewer radiofrequency deliveries for SVC isolation than the Non-Block group ( $4.2 \pm 0.9$ vs. $10.2 \pm 2.8$ times; $P<0.0001)$. The isolated SVC area was larger in the Block group (15.7 \pm 3.7 vs. $\left.10.5 \pm 3.1 \mathrm{~cm}^{2} ; \mathrm{P}<0.0001\right)$.
\end{abstract}

Conclusions: We found that approximately half of patients with AF had a diagonal line of block at the RA-SVC junction that could be utilized to isolate the SVC with fewer radiofrequency deliveries.

Key Words: Conduction block; High-resolution mapping; RHYTHMIA mapping system; Superior vena cava

$\mathbf{P}$ revious studies have demonstrated that atrial fibrillation (AF) is most often initiated by ectopic firing from the pulmonary veins (PVs), resulting in electrical disconnection of the PVs from the left atrium (LA) becoming the cornerstone of AF treatment. ${ }^{1-4}$ However, ectopy from non-PV foci can also be an important source of AF.$^{5-7}$ Because the superior vena cava (SVC) is one of the most common sites of non-PV foci, performing electrical SVC isolation in addition to PV isolation (PVI) can improve the clinical outcome of AF ablation..$^{89}$ However, little is known about the electrophysiological properties of the junctional area between the right atrium (RA) and SVC.

Recently, a new 3D mapping system (RHYTHMIA, Boston Scientific, Cambridge, MA, USA) that allows rapid and ultra-high-resolution electroanatomic mapping was launched. ${ }^{10-12}$ Although some previous publications have reported the feasibility of this technology for mapping atrial arrhythmias, including for PVI, ${ }^{13,14}$ the utility of this system for SVC isolation has not been fully evaluated.

The aim of this study was to assess RA-SVC conduction patterns and to determine the ideal procedure for electrically isolating the SVC from the RA using this high-resolution mapping system.

\section{Methods}

\section{Patient Population}

In this prospective study, we performed RA-SVC activation mapping using the RHYTHMIA system in consecutive patients who underwent PVI at Yokosuka Kyosai Hospital between November 2016 and April 2017. Patients who had received any ablation procedures prior to this study were excluded. Written informed consent was obtained from each patient prior to the procedure. This study was approved by the institutional ethics boards.

\section{PVI}

All patients underwent PVI before RA-SVC mapping. Antiarrhythmic drugs were withdrawn for at least 5 half-lives before the procedure. An activated clotting time of 300-400 s was maintained with a continuous infusion of heparin during the procedure. In the patients whose basic rhythm was AF, the AF was terminated using an internal cardioversion system (BeeAT, Japan Lifeline, Tokyo, Japan). The strategy of PVI has been described previously. ${ }^{15,16}$ Briefly, after a trans-septal puncture, 2 circular mapping catheters were placed in the superior and inferior PVs, and the left and right ipsilateral PVs were circumferentially

Received December 7, 2017; revised manuscript received April 19, 2018; accepted April 25, 2018; released online June 7, 2018 Time for primary review: 32 days

The Cardiovascular Center, Yokosuka Kyosai Hospital, Yokosuka (Y.T., A.T., T.T., J.N., K.T., H.H.); The Heart Rhythm Center, Tokyo Medical and Dental University, Tokyo (M.G., K.H.), Japan

Mailing address: Yasuaki Tanaka, MD, The Cardiovascular Center, Yokosuka Kyosai Hospital, 1-16 Yonegahamadori, Yokosuka 238-8558, Japan. E-mail: yasuaki@mxk.mesh.ne.jp

ISSN-1346-9843 All rights are reserved to the Japanese Circulation Society. For permissions, please e-mail: cj@j-circ.or.jp 


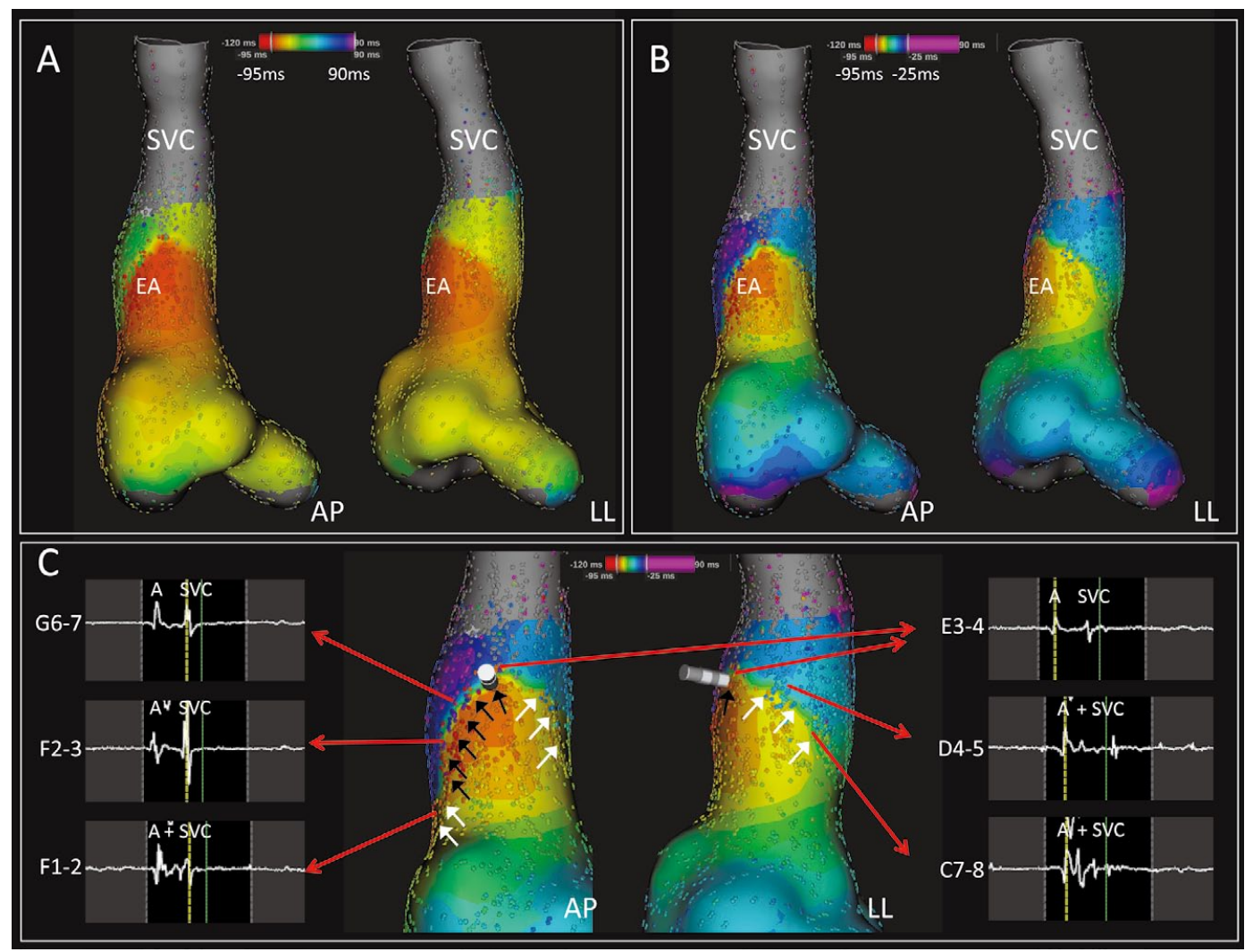

Figure 1. Software set-up process for visualization of conduction in the right atrium-superior vena cava junction region. (A) We shifted the color bar so that the site of EA fell within the red zone of the spectrum. (B) The remaining 6 colors of the spectrum then aligned naturally with successive approximately $10 \mathrm{~ms}$ increments after the EA, ending in violet (i.e., activation 60-70 ms after EA was represented by the color violet). (C) Conduction block was defined as the line where the spectrum spanned $\geq 3$ colors within a short distance $(<3 \mathrm{~mm})$, corresponding to double potentials with $\geq 30 \mathrm{~ms}$ separation in electrograms recorded along the line. Black arrows point to a line of complete conduction block, white arrows to incomplete conduction block. AP, anteroposterior view; EA, earliest activation; LL, left lateral view; SVC, superior vena cava.

ablated under fluoroscopic and electrophysiological guidance. The endpoint was the elimination or dissociation of $\mathrm{PV}$ potentials.

\section{Electroanatomic Mapping of the RA-SVC Junction}

After completion of PVI, RA-SVC angiography was performed by manual injection. The junction of the convex RA wall and the straight SVC wall on the angiogram was defined as the radiological RA-SVC junction. ${ }^{17}$ Electroanatomic maps of the region around the radiologic RA-SVC junction were created using the RHYTHMIA system with the INTELLAMAP ORION basket catheter (Boston Scientific). A diagnostic catheter was placed in the inferior vena cava to serve as an indifferent unipolar electrode. The RHYTHMIA system has previously been described in detail.10,12 Data acquisition during sinus rhythm was automated with the following beat acceptance criteria: (1) sinus cycle length (CL) stability $( \pm 50 \mathrm{~ms})$; (2) time stability of a reference electrogram positioned at the coronary sinus; (3) beat-to-beat electrogram consistency; and (4) $\geq 3$ consecutive beats with similar electrogram morphology and timing. The criteria are set by the operator before the start of mapping and remain fixed even if the sinus CL alters during mapping. The electrograms of the accepted beats are programmed to be included in the activation map only when the electrode is within $2 \mathrm{~mm}$ (projection distance setting) of the endocardial surface. Complete electrical silence was defined as activity $<0.03 \mathrm{mV}$ (confidence mask setting) and depicted by a gray color on the map. After data acquisition, individual activation at a selected location was reviewed by sliding the cursor over the location.

Mapping was considered complete when the morphological aspect of the map appeared to reflect the anatomy of the RA and SVC, and the electrical aspect of the map displayed a uniform distribution of potentials. The SVC sleeve length was measured as the distance between the radiologic RA-SVC junction and the highest level of SVC sleeve (Figure S1).

We conceptually defined the sinus node as the region displaying the earliest activation (EA) in the map, which was in turn defined as the time and site of the earliest unipolar electrogram displaying a "QS" pattern. ${ }^{18}$ Activation was defined as unicentric if 1 site of EA was present, and as multicentric if $\geq 2$ EA with activation time difference $\leq 5 \mathrm{~ms}$ separated by a distance $>10 \mathrm{~mm}$ was observed. 


\begin{tabular}{|c|c|c|c|c|}
\hline & $\begin{array}{l}\text { Overall } \\
(n=113)\end{array}$ & $\begin{array}{l}\text { Block group } \\
\quad(n=56)\end{array}$ & $\begin{array}{l}\text { Non-block group } \\
(n=57)\end{array}$ & $P$ value \\
\hline Age (years) & $62.8 \pm 11.5$ & $65.8 \pm 10.5$ & $59.8 \pm 11.8$ & 0.0055 \\
\hline Sex: male (n) & $80(71 \%)$ & $41(73 \%)$ & $39(68 \%)$ & 0.68 \\
\hline Hypertension (n) & $53(47 \%)$ & $28(50 \%)$ & $25(44 \%)$ & 0.57 \\
\hline Diabetes (n) & $10(9 \%)$ & $6(11 \%)$ & $4(7 \%)$ & 0.53 \\
\hline TIA/stroke (n) & $3(3 \%)$ & $2(4 \%)$ & $1(2 \%)$ & 0.62 \\
\hline Heart failure (n) & $8(7 \%)$ & $4(7 \%)$ & $4(7 \%)$ & 1.00 \\
\hline Vascular disease (n) & $2(2 \%)$ & $2(4 \%)$ & 0 & 0.24 \\
\hline $\mathrm{CHA}_{2} \mathrm{DS}_{2}$-VASC score & $2[1-2.5]$ & $2[1-3]$ & $1[0-2]$ & 0.09 \\
\hline Duration from initial AF diagnosis (months) & $70.6 \pm 90.8$ & $69.7 \pm 85.7$ & $71.5 \pm 96.9$ & 0.92 \\
\hline Paroxysmal AF (n) & 67 (59\%) & $31(55 \%)$ & $36(63 \%)$ & 0.45 \\
\hline LVEF (\%) & $63.1 \pm 6.9$ & $63.0 \pm 6.5$ & $63.2 \pm 7.3$ & 0.91 \\
\hline Left atrial diameter (mm) & $39.7 \pm 5.4$ & $39.9 \pm 5.5$ & $39.5 \pm 5.4$ & 0.74 \\
\hline Sinus cycle length (ms) & $917 \pm 227$ & $957 \pm 227$ & $877 \pm 221$ & 0.059 \\
\hline
\end{tabular}

$\mathrm{AF}$, atrial fibrillation; LVEF, left ventricular ejection fraction; TIA, transient ischemic attack.

\section{Setting the Timing Frame (Color Bar) in the Activation Map}

To optimize visualization of conduction at the RA-SVC junction, we took special care in how we set the timing frames (color bar) in the activation color maps. They were set in the following order. (1) Reference time $(0 \mathrm{~ms})$ set as the sharpest deflection recorded by the bipolar CS electrode. The software then automatically assigned colors to the time range of the earliest activity in the map to the latest (e.g., from $-120 \mathrm{~ms}$ to $+90 \mathrm{~ms}$ ). It is possible to shift the color bar relative to the time range, so we did this to ensure that the EA fell within the red part of the spectrum (Figure 1A). (2) The remaining 6 colors of the spectrum then aligned naturally with successive approximately $10 \mathrm{~ms}$ increments after the EA, ending in violet (i.e., activation $60-70 \mathrm{~ms}$ after the EA was represented by the color violet) (Figure 1B). Conduction block was defined as the line where the spectrum spanned $\geq 3$ colors within a short distance, corresponding to double potentials with $\geq 30 \mathrm{~ms}$ separation in electrograms recorded along the line (Figure 1C, black arrows).

The way we set the timing bar allowed us to clearly visualize lines of block, which made it easy for us to subdivide the patients into 2 groups by the presence (Block group) or absence (Non-block group) of a conduction block at the RA-SVC junction.

\section{SVC Isolation}

After the mapping, provocation of non-PV foci was attempted with administration of high dose isoproterenol $(\leq 20 \mu \mathrm{g} / \mathrm{min})$. We also looked for electrograms after electrical cardioversion of pacing-induced AF. Only when foci in the SVC initiating AF were identified was electrical isolation of the SVC from the RA performed using the mapping data. (1) If a conduction block line was observed at the RA-SVC junction, point-by-point RF applications were delivered along the line connecting the open ends of the conduction block line. The result was a circumferential line of block around the SVC. (2) In patients without a conduction block line, SVC isolation was performed at approximately $5 \mathrm{~mm}$ above the radiologic RA-SVC junction. To avoid sinus node injury, we made sure that the ablation sites were far from the EA identified by the RHYTHMIA system. In both methods, RF energy of 20-30 W with $17 \mathrm{~mL} / \mathrm{min}$ of saline irrigation and a maximum temperature of $42^{\circ} \mathrm{C}$ were delivered using an open irrigation catheter (FlexAbility, St. Jude Medical, Minneapolis, MN, USA). The endpoint of SVC isolation was complete elimination of SVC potentials. To avoid phrenic nerve injury, high current stimulation (bipolar pacing, $10 \mathrm{~mA}$ at $2 \mathrm{~ms}$ ) along the lateral portion of the RA-SVC junction and careful observation of diaphragmatic motion during RF application were performed. The positions where the phrenic nerve was captured were plotted on 3D mapping. To confirm SVC isolation, activation mapping was performed during sinus rhythm using the RHYTHMIA system again. The size of the isolated area was measured to compare the voltage mapping before and after SVC isolation.

\section{Statistical Analysis}

Data are presented as the mean \pm standard deviation, percentage, or number, as appropriate. Differences were assessed using an unpaired 2-tailed t test for normally distributed continuous variables, Mann-Whitney test for skewed continuous variables, and the chi-square test for nominal variables.

\section{Results}

\section{Patients' Characteristics}

We performed mapping of the RA-SVC junction in 116 patients. After excluding 3 patients with no SVC potentials, a total number of 113 patients ( 80 males) with symptomatic AF, who underwent ultra-high-resolution mapping of RA-SVC conduction during sinus rhythm, were assessed: $67(59 \%)$ patients had paroxysmal $\mathrm{AF}$ and the remaining $46(41 \%)$ had non-paroxysmal AF. The mean age was $62.8 \pm 11.5$ years, mean left ventricular ejection fraction was $63.1 \pm 6.9 \%$, and mean LA diameter was $39.7 \pm 5.4 \mathrm{~mm}$. These and other data are summarized in Table 1.

\section{Procedural Data}

RA-SVC maps were successfully obtained using the RHYTHMIA system without complications. A total of $203 \pm 148$ beats were accepted per map. From the included 


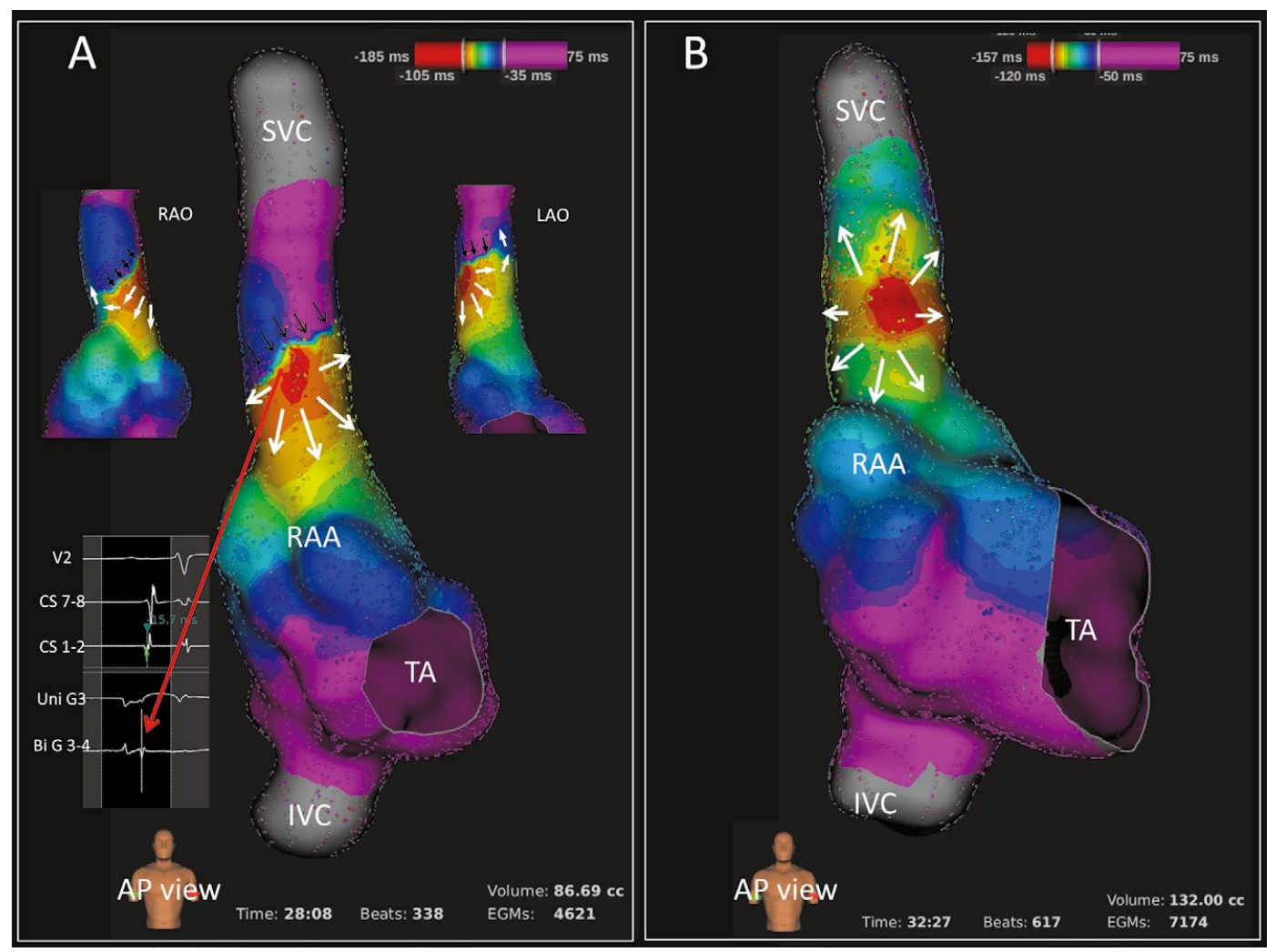

Figure 2. The 2 types of conduction pattern between the right atrium (RA) and superior vena cava (SVC). (A) Typical case of conduction block: the line of conduction block interfered with immediate spread of RA activity into the SVC. Electrogram recordings from the conduction block line showed double potentials representing an atrial potential followed by a sharp SVC potential. The central map shows the anteroposterior view (AP), and on either side, the RAO and LAO views. (B) Patient without conduction block (Non-block group): activation from the sinus node spreads concentrically. Black arrows: conduction block, White arrows: trace of propagation. IVC, inferior vena cava; LAO, left anterior oblique view; RAA, right atrial appendage; RAO, right anterior oblique view; TA, tricuspid annulus.

beats, a total of $3,176 \pm 1,899$ electrograms were recorded. Manual annotation, although available, was not necessary in any patient. The mean mapping time was $10.8 \pm 5.5 \mathrm{~min}$.

\section{Location of Sinus Node}

The mean sinus CL at the beginning of RA-SVC mapping was $917 \pm 227 \mathrm{~ms}$ (range 498-1,621 ms). The EA sites were unicentric in 80 patients, and multicentric in 33 patients. The unicentric EAs were located at the anterior $(n=41)$, anterolateral $(n=23)$, lateral $(n=9)$ and posterolateral wall $(n=7)$ of the high RA. The multicentric EA were all bicentric: anterior+lower anterolateral $(n=26)$, anterior+lower lateral $(n=3)$, and anterolateral+lower lateral $(n=4)$.

\section{Conduction Pattern Around the RA-SVC Junction}

A spontaneous conduction block line between the RA and the SVC was observed in 56 patients (49.5\%: Block group (Figure 2A)). Electrogram recordings from the conduction block line showed double potentials representing an atrial potential followed by a sharp SVC potential. The conduction block lines were classified into 3 types according to the shape of the block line. (1) Type I (straight block line): observed in 23 patients (41.1\%; Figure 3A). An oblique straight line of conduction block running from the lower lateral wall to the higher anterior wall interfered with immediate activation spread into the SVC. (2) Type J (reverse $\mathrm{J}$ shape block line): the conduction block line ran from the lower posterolateral wall to the higher or midseptal wall in an arc with its highest portion at the anterior wall. This was observed in 29 patients $(51.8 \%$; Figure 3B). (3) Type U (reverse U shape block line): 4 patients (7.1\%) had a block line that extended from the lower posterior to lower posteroseptal via higher anterior wall around the RA-SVC junction (Figure 3C). In a different projection, a conduction gap could be seen at the lower posterior part of the junction. Although there were variations in the conduction block shape, the diagonal portion of the block line from lower lateral wall to higher anterior wall was common to all of them. The prevalence of each block type is shown in Figure 3D. The length of the line of conduction block varied among patients (median $18.8 \mathrm{~mm}$, range 9.4 $59.9 \mathrm{~mm}$ ). The mean lengths of the block line in each type are shown in Figure 3E.

In 57 patients, conduction block was not observed (50.5\%: Non-block group, Figure 2B). With respect to their baseline clinical characteristics, these patients were younger than those in the Block group $(59.8 \pm 11.8$ years vs. $65.8 \pm 10.5$ years; $\mathrm{P}<0.05)$. Otherwise, there were no signifi- 

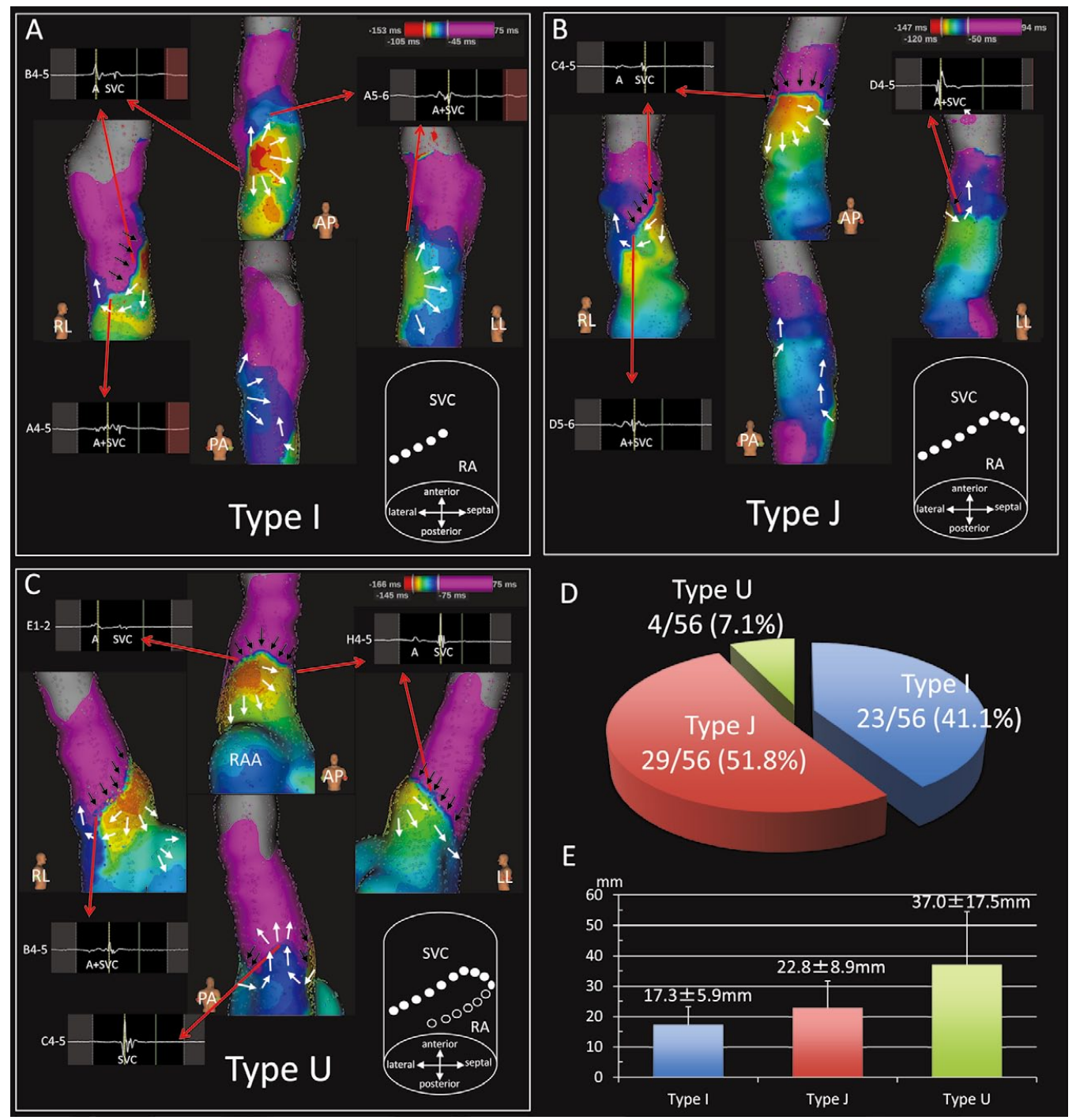

Figure 3. Classification of conduction block between the RA and SVC according to shape of the block line. (A) Type I (straight block line): straight line of conduction block running obliquely from the lower lateral wall to higher anterior wall. (B) Type $J$ (reverse $\mathrm{J}$ shape block line): conduction block line runs from the lower posterolateral wall to the higher or mid-septal wall via its highest point on the anterior wall. $(\mathbf{C})$ Type $U$ (reverse $U$ shape block line): block line extends the lower posterior to lower posteroseptal via the higher anterior wall around the RA-SVC junction. Although there are variations in the conduction block shape, the diagonal portion of the block line from the lower lateral wall to higher anterior wall is common to all of them. (D) Pie chart showing prevalence of each block type. (E) Length of the line of conduction block by conduction block type. PA, postero-anterior view; RL, right lateral view. Other abbreviations as in Figures 1,2.

cant differences between the 2 groups. The sinus CL had a tendency to be longer in the Block group $(\mathrm{P}=0.059)$. The data are summarized in Table $\mathbf{1}$.

\section{SVC Isolation}

Ectopy originating from the SVC spontaneously initiated $\mathrm{AF}$ in 43 patients $(38.1 \%)$, so electrical SVC isolation was performed in those patients. Conduction block line was observed in 22 patients (i.e., in approximately half of the Block group). As described in the Methods section, Block group patients received point-by-point RF applications that were delivered to connect the 2 open ends of the conduction block line. RF applications were not necessary along the block line. Typical ablation points are shown in Figure 4A and Figure 5. In contrast, in the patients without conduction block line $(n=21)$, circumferential RF applications were needed for complete elimination of SVC potentials (Figure 4B). Therefore, the number of RF deliveries, the total RF energy and procedural duration until SVC isolation in the Block group patients were significantly less than in the Non-block group patients $(4.2 \pm 0.9$ times vs. $10.2 \pm 2.8$ times; $\mathrm{P}<0.0001 ; 5,042 \pm 1,581 \mathrm{~J}$ vs. $10,582 \pm 2,335$ $\mathrm{J}, \mathrm{P}<0.0001 ; 3.3 \pm 1.2$ vs. $10.2 \pm 2.8 \mathrm{~min}, \mathrm{P}<0.0001$, respectively). After complete elimination of SVC potentials, dissociated activity within the SVC was observed in 18 of the 22 Block group patients. There was no significant differ- 


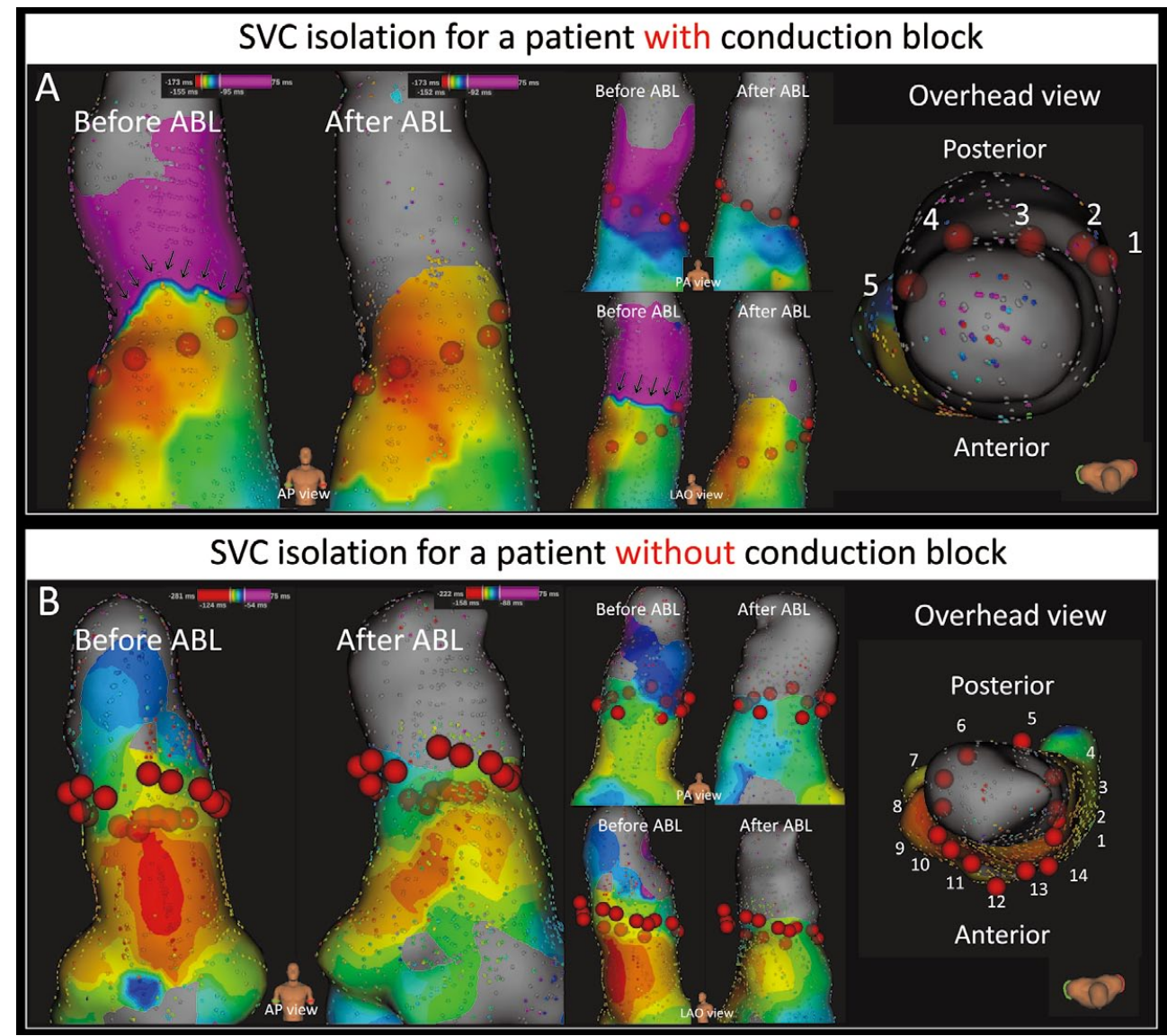

Figure 4. SVC isolation for patients with $(\mathbf{A})$ and without (B) conduction block. In the patient from the Block group (A), point-by-point RF applications were delivered to connect the open ends of the conduction block line. In the PA view, the RF application points are seen through the SVC. On the other side, RF application was not required along the pre-existing block line. In this patient, SVC isolation was achieved with only 5 radiofrequency applications, because ABL was unnecessary along the block line. In the patient from the Non-block group (B), circumferential RF applications were needed for complete elimination of SVC potentials. A total of 14 radiofrequency applications were required. ABL, ablation. Other abbreviations as in Figures 2,3.

ence between the Block and Non-Block groups in the SVC sleeve length (Block vs. Non-block: $43.3 \pm 8.0 \mathrm{~mm}$ vs. $43.7 \pm 11.7 \mathrm{~mm}, \mathrm{P}=0.911)$. Nevertheless, the size of the isolated area in the Block group was larger than that in the Non-block group $\left(15.7 \pm 3.7 \mathrm{~cm}^{2}\right.$ vs. $\left.10.5 \pm 3.1 \mathrm{~cm}^{2}, \mathrm{P}<0.0001\right)$. These data are summarized in Table 2.

In Non-block patients, although significant sinus node injury (sinus pause $>3 \mathrm{~s}$, significant sinus bradycardia, or persistent slow junctional rhythm) was never observed, the sinus CL increased by more than $10 \%$ during RF deliveries on the anterior wall. In contrast, no significant change was observed in sinus CL in the patients who had a conduction block line because there was no necessity for RF delivery near the sinus node.

The sites of phrenic nerve capture were frequently located on the intended ablation line in the Non-block patients (16/21 patients: $76 \%$, Figure 6A). Despite of careful monitoring of diaphragmatic motion during RF applications, persistent phrenic nerve injury occurred in 2 Non-block patients. Impaired right diaphragmatic movement in these patients completely recovered within 2 months after ablation. On the other hand, phrenic nerve capture was rarely observed (5/22 patients: $23 \%$, Figure 6B) in the patients with a conduction block line, because the lateral part of the ablation line was lower than that in the Non-block patients. This is likely why phrenic nerve injury was not observed in the Block group.

\section{Discussion}

The main findings of this study were: (1) using ultra highresolution mapping enabled the creation of detailed activation maps of the sinus node and RA-SVC junction during sinus rhythm; (2) a spontaneous conduction block line was detected just above the sinus node in approximately half of the patients; and (3) when isolating the SVC, RF delivery count and risk of sinus node and phrenic nerve injury were reduced in the patients with an identifiable conduction block line.

Several previous animal studies have reported the feasibility of the RHYTHMIA electroanatomic mapping system and basket catheter for RA mapping. In those animal studies, it was demonstrated that the mini-basket catheter could rapidly acquire high-resolution electroanatomic 


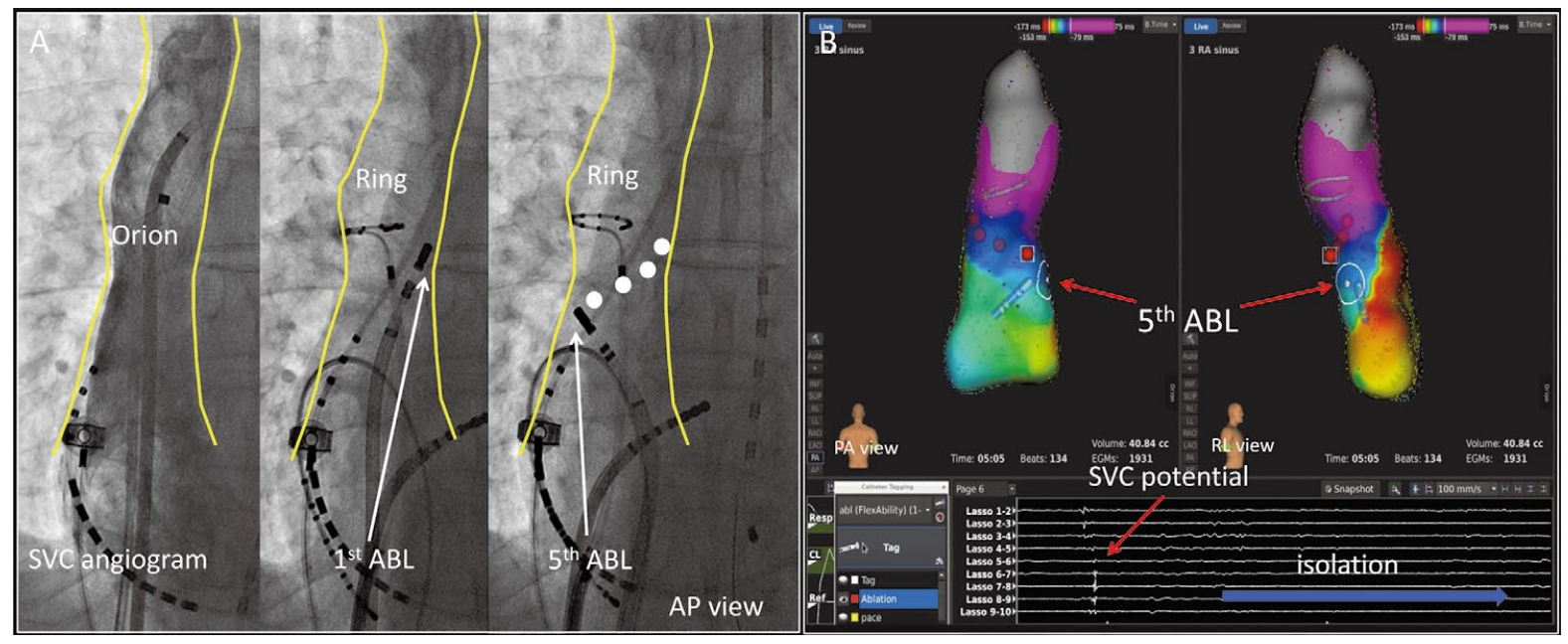

Figure 5. Examples of $A B L$ points. (A) Fluoroscopic image of the RA-SVC junction during SVC isolation. Before mapping and $\mathrm{ABL}$, the SVC angiogram in all patients was taken with manual injection of contrast media. In this patient with conduction block, the $A B L$ line courses diagonally. The ABL points are shown as white dots. (B) RHYTHMIA map of ABL points. In the same patient as in (A), SVC isolation is achieved with only 5 radiofrequency applications because of the pre-existing conduction block. Abbreviations as in Figures 2-4.

\begin{tabular}{|c|c|c|c|}
\hline & $\begin{array}{l}\text { Block group } \\
\quad(n=22)\end{array}$ & $\begin{array}{l}\text { Non-block group } \\
(n=21)\end{array}$ & $P$ value \\
\hline SVC sleeve length (mm) & $43.3 \pm 8.0$ & $43.7 \pm 11.7$ & 0.911 \\
\hline Procedural time to SVC isolation ( $\mathrm{min}$ ) & $3.3 \pm 1.2$ & $7.8 \pm 2.6$ & $<0.0001$ \\
\hline No. of RF deliveries & $4.2 \pm 0.9$ & $10.2 \pm 2.8$ & $<0.0001$ \\
\hline Total RF energy (J) & $5,042 \pm 1,581$ & $10,582 \pm 2,335$ & $<0.0001$ \\
\hline Isolated area $\left(\mathrm{cm}^{2}\right)$ & $15.7 \pm 3.7$ & $10.5 \pm 3.1$ & $<0.0001$ \\
\hline
\end{tabular}

$\mathrm{RF}$, radiofrequency; SVC, superior vena cava.

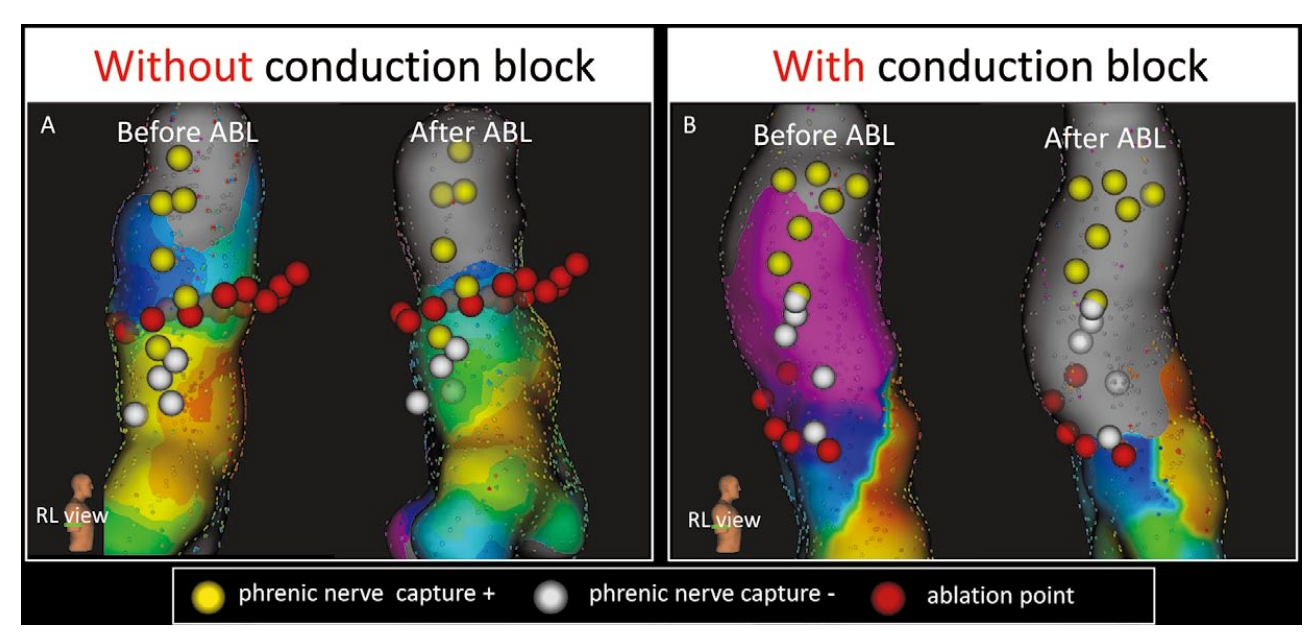

Figure 6. Sites of phrenic nerve capture were $(\mathbf{A})$ frequently located on the intended ABL line in the Non-block patients (16/21 patients: $76 \%$ ). (B) In this patient from the Block group, sites of phrenic nerve capture are superior to the line of block. The phrenic nerve was rarely captured (5/22 patients: $23 \%)$ in patients with a conduction block line. Yellow dots: sites of phrenic nerve capture; White dots: sites without phrenic nerve capture; Red dots: ABL points. ABL, ablation; RL, right lateral view. 
maps. Nakagawa et al created a canine RA linear lesion model to test whether this system could map a complex activation pattern in the atrium and reported that this system could accurately recreate the geometry and localize the line of block and electrical gap. ${ }^{10}$ Another study showed that detailed endocardial assessment of sinus node activation in a swine model using RHYTHMIA was feasible and that EA sites were located parallel to the crista terminalis around the RA-SVC junction. ${ }^{11}$ Our clinical study confirmed the feasibility of using the RHYTHMIA system to visualize the detailed activation of the sinus node and conduction between the RA and SVC during sinus rhythm.

Regarding the location of the EA sites of the sinus node, there is a lot of individual variation. Boineau et al using traditional mapping methods reported unifocal nodal and extranodal sinus origins in addition to multicentric origins (simultaneous activation at disparate sites) over 2-4 widely distributed pacemaker sites. ${ }^{19}$ In contrast, high-density, non-contact mapping for single beat analysis has not found multicentric (simultaneous) EA. However, the intraindividual beat-to-beat EA location was variable in the range of up to $41 \mathrm{~mm} .{ }^{20}$ In our maps, multicentric, or more accurately, bicentric activation, was observed in approximately $30 \%$ of cases. However, because the maps were a composite of several hundred beats obtained from contact mapping, we cannot say whether multicentric EA resulted from simultaneous activation or not. Multicentric activation might result from beat-to-beat shifts of EA-dependent variability in the CL. Either way, identifying the location of the sinus node complex helped us avoid sinus node injury during SVC isolation.

The SVC is a well-known and important focus triggering AF. 8,9 Although electrical isolation of arrhythmogenic SVC foci and the RA is commonly performed in AF ablation, the electrophysiologic properties of the SVC and RA-SVC junction have received little research attention. Some human histologic studies show that myocardial sleeves extend from the RA into the SVC for up to $2-5 \mathrm{~cm},{ }^{21}$ and are recognized in $76 \%$ of SVCs, although the RA-SVC myocardial connection is discontinuous in most cases.22 Goya et al reported identifying breakthroughs between the SVC and RA and that they could be disconnected by ablating the earliest activated SVC potential during sinus rhythm in a point-by-point fashion using 3D electroanatomic mapping, rather than circumferential ablation. ${ }^{17}$ However, discrimination of SVC electrograms from RA electrograms recorded by a multipolar catheter placed on the RA-SVC junction is difficult because the potentials often appear to be fused together. Although there are some case reports showing that non-contact mapping can localize the electrical breakthroughs at the RA-SVC junction and guide ablation of SVC isolation, 23,24 non-contact activation maps cannot depict propagation accurately enough to guide ablation in most cases. ${ }^{25}$ In our study, the RA-SVC conduction properties were visualized as a conduction block rather than as breakthroughs, and this conduction block was present in approximately half of the cases.

In a recent clinical study using RHYTHMIA for SVC mapping and ablation, Yamashita et $\mathbf{a l}^{\mathbf{2 6}}$ reported that in 32 of 36 patients atrial activation from the sinus node conducted in a superior and clockwise direction from the sinus node, a finding that the authors referred to as spiral activation. In the remaining 4 patients, activation was superior and either vertical or counterclockwise. Those findings are in agreement with ours, and are presumably caused by the diagonal block lines just above the sinus node that we have described. The focus of our study differed from that of the Yamashita study because we were interested in the location and prevalence of identifiable conduction block. We classified the different patterns of block according to their shape and also described how these patterns could be utilized to make decisions regarding ablation lines. Namely, we determined that if a block line was observed, RF application along the block line was unnecessary for SVC isolation, leading to a reduced number of RF deliveries.

Although we found 3 types of conduction block shape, the diagonal portion from the lower lateral wall to the higher anterior wall was common to all of them. Because all previous clinical studies have assumed that the RA-SVC junction is a horizontal line, $\mathbf{5 , 6 , 1 7 , 2 5}$ this is another new finding from our study. We believe that this diagonal block line constitutes a functional (electrical) border between the RA and SVC and that the block line corresponds to the superior edge of the crista terminalis based on the anatomic location. Because we did not perform additional activation mapping during pacing within the SVC, because of time constraints, exit block (the block of conduction from the SVC to RA) was not studied. However, dissociated electrograms within the SVC after its isolation strongly suggested the existence of bidirectional block. We also noted that patients with conduction block line were older $(\mathrm{P}<0.05)$, leading us to conjecture that conduction block may advance with age, although other clinical parameters that could be associated with age did not differ between the 2 groups.

There are 2 major complications during SVC isolation that must be avoided. One is sinus node injury, which usually occurs if RF energy is applied too close to the sinus node complex. High-resolution mapping to identify EA in the sinus node region helps to reduce sinus node injury. We found identification of the conduction block line in the patients who had them very useful, because almost all block lines were located just above the sinus node complex, allowing us to forego unnecessary RF delivery near the sinus node. Right phrenic nerve injury is the other and most frequently observed major complication. The right phrenic nerve runs close to the superior part of the SVC. The phrenic nerve was rarely captured on the ablation line in patients with conduction block, because in the patients with a conduction block, our ablation line was located in the lower part of the lateral SVC. Gianni et al recently reported that SVC isolation could be completed by targeting its septal segment and sites of EA lower in the RA posterior wall when SVC isolation with a standard ablation approach was impossible because of phrenic nerve capture. ${ }^{27}$ As a result, their ablation points correspond to our SVC isolation line in patients with a conduction block line.

\section{Study Limitations}

There were several limitations to the present study. The study population, especially that of the patients who underwent SVC isolation, was relatively small. Further evaluation with a larger number of patients is required. The patient population of this study was limited to those without structural heart disease or systolic dysfunction. Therefore, we cannot extend the results of this study to all patients with AF. Because we have yet to conduct a followup study of the patients receiving extensive SVC isolation, 
the long-term safety of this treatment is unclear.

\section{Conclusions}

We found that approximately half of the patients with $\mathrm{AF}$ had a diagonal line of block at the RA-SVC junction that could be utilized to isolate the SVC with fewer radiofrequency deliveries.

\section{Disclosures}

The authors have no relationships with industry that could pose a conflict of interest with regard to this study.

\section{References}

1. Haïssaguerre M, Jaïs P, Shah DC, Takahashi A, Hocini M, Quiniou G, et al. Spontaneous initiation of atrial fibrillation by ectopic beats originating from the pulmonary veins. $N$ Engl $J$ Med 1998; 339: 659-666.

2. Haïssaguerre M, Jaïs P, Shah DC, Garrigue S, Takahashi A, Lavergne $\mathrm{T}$, et al. Electrophysiological end point for catheter ablation of atrial fibrillation initiated from multiple pulmonary venous foci. Circulation 2000; 101: 1409-1417.

3. Arentz T, Weber R, Bürkle G, Herrera C, Blum T, Stockinger J, et al. Small and large isolation areas around the pulmonary veins for treatment of atrial fibrillation?: Results from a prospective randomized study. Circulation 2007; 115: 3057-3063.

4. Liu X, Dong J, Mavrakis HE, Hu F, Long D, Fang D, et al. Achievement of pulmonary vein isolation in patients undergoing circumferential pulmonary vein ablation: A randomized comparison between two different isolation approaches. J Cardiovasc Electrophysiol 2006; 17: 1263-1270.

5. Tsai CF, Tai CT, Hsieh MH, Lin WS, Yu WC, Ueng KC, et al. Initiation of atrial fibrillation by ectopic beats originating from the superior vena cava: Electrophysiological characteristics and results of radiofrequency ablation. Circulation 2000; 102: 67-74.

6. Lin WS, Tai CT, Hsieh MH, Tsai CF, Lin YK, Tsao HM, et al. Catheter ablation of paroxysmal atrial fibrillation initiated by non-pulmonary vein ectopy. Circulation 2003; 107: 3176-3183.

7. Shah D, Haissaguerre M, Jais P, Hocini M. Nonpulmonary vein foci: Do they exist? Pacing Clin Electrophysiol 2003; 26: 16311635 .

8. Arruda M, Mlcochova H, Prasad SK, Kilicaslan F, Saliba W, Patel D, et al. Electrical isolation of the superior vena cava: An adjunctive strategy to pulmonary vein antrum isolation improving the outcome of AF ablation. J Cardiovasc Electrophysiol 2007; 18: $1261-1266$.

9. Corrado A, Bonso A, Madalosso M, Rossillo A, Themistoclakis $\mathrm{S}$, Di Biase L, et al. Impact of systematic isolation of superior vena cava in addition to pulmonary vein antrum isolation on the outcome of paroxysmal, persistent, and permanent atrial fibrillation ablation: Results from a randomized study. J Cardiovasc Electrophysiol 2010; 21: 1-5.

10. Nakagawa H, Ikeda A, Sharma T, Lazzara R, Jackman WM. Rapid high resolution electroanatomic mapping: Evaluation of a new system in a canine atrial linear lesion model. Circ Arrhythm Electrophysiol 2012; 5: 417-424.

11. Bollmann A, Hilbert S, John S, Kosiuk J, Hindricks G. Insights from preclinical ultra high-density electroanatomical sinus node mapping. Europace 2015; 17: 489-494.

12. Tanaka Y, Genet M, Chuan Lee L, Martin AJ, Sievers R, Gerstenfeld EP. Utility of high-resolution electroanatomic mapping of the left ventricle using a multispline basket catheter in a swine model of chronic myocardial infarction. Heart Rhythm 2015; 12: $144-154$

13. Anter E, McElderry TH, Contreras-Valdes FM, Li J, Tung P,
Leshem E, et al. Evaluation of a novel high-resolution mapping technology for ablation of recurrent scar-related atrial tachycardias. Heart Rhythm 2016; 13: 2048 -2055.

14. Anter E, Tschabrunn CM, Contreras-Valdes FM, Li J, Josephson ME. Pulmonary vein isolation using the Rhythmia mapping system: Verification of intracardiac signals using the Orion mini-basket catheter. Heart Rhythm 2015; 12: 1927-1934.

15. Kuwahara T, Takahashi A, Kobori A, Miyazaki S, Takahashi Y, Takei A, et al. Safe and effective ablation of atrial fibrillation: Importance of esophageal temperature monitoring to avoid periesophageal nerve injury as a complication of pulmonary vein isolation. J Cardiovasc Electrophysiol 2009; 20: 1-6.

16. Takigawa M, Kuwahara T, Takahashi A, Okubo K, Takahashi Y, Nakashima E, et al. Simultaneous isolation of superior and inferior pulmonary veins on both the left and right sides could yield better outcomes in patients with paroxysmal atrial fibrillation. Europace 2015; 17: 732-740.

17. Goya M, Ouyang F, Ernst S, Volkmer M, Antz M, Kuck KH. Electroanatomic mapping and catheter ablation of breakthroughs from the right atrium to the superior vena cava in patients with atrial fibrillation. Circulation 2002; 106: 1317-1320.

18. Higa S, Tai CT, Lin YJ, Liu TY, Lee PC, Huang JL, et al. Focal atrial tachycardia: New insight from noncontact mapping and catheter ablation. Circulation 2004; 109: 84-91.

19. Boineau JP, Canavan TE, Schuessler RB, Cain ME, Corr PB, Cox JL. Demonstration of a widely distributed atrial pacemaker complex in the human heart. Circulation 1988; 77: 1221-1237.

20. Stiles MK, Brooks AG, Roberts-Thomson KC, Kuklik P, John B, Young GD, et al. High-density mapping of the sinus node in humans: Role of preferential pathways and the effect of remodeling. J Cardiovasc Electrophysiol 2010; 21: 532-539.

21. Hashizume H, Ushiki T, Abe K. A histological study of the cardiac muscle of the human superior and inferior venae cavae. Arch Histol Cytol 1995; 58: 457-464.

22. Kholova I, Kautzner J. Morphology of atrial myocardial extensions into human caval veins: A postmortem study in patients with and without atrial fibrillation. Circulation 2004; 110: $483-$ 488.

23. Liu TY, Tai CT, Lee PC, Hsieh MH, Higa S, Ding YA, et al. Novel concept of atrial tachyarrhythmias originating from the superior vena cava: Insight from noncontact mapping. $J$ Cardiovasc Electrophysiol 2003; 14: 533-539.

24. Weiss C, Willems S, Rostock T, Risius T, Ventura R, Meinertz $\mathrm{T}$. Electrical disconnection of an arrhythmogenic superior vena cava with discrete radiofrequency current lesions guided by noncontact mapping. Pacing Clin Electrophysiol 2003; 26: $1758-$ 1761 .

25. Fukumoto K, Takatsuki S, Kimura T, Nishiyama N, Tanimoto $\mathrm{K}$, Aizawa Y, et al. Electrophysiological properties of the superior vena cava and venoatrial junction in patients with atrial fibrillation: Relevance to catheter ablation. J Cardiovasc Electrophysiol 2014; 25: 16-22.

26. Yamashita S, Tokuda M, Isogai R, Tokutake K, Yokoyama K, Narui R, et al. Spiral activation of the superior vena cava: The utility of ultra-high-resolution mapping for caval isolation. Heart Rhythm 2018; 15: 193-200.

27. Gianni C, Sanchez JE, Mohanty S, Trivedi C, Della Rocca DG, $\mathrm{Al}$-Ahmad A, et al. Isolation of the superior vena cava from the right atrial posterior wall: A novel ablation approach. Europace, doi:10.1093/europace/eux262.

\section{Supplementary Files}

\section{Supplementary File 1}

Figure S1. Method used to measure the SVC sleeve length.

Please find supplementary file(s);

http://dx.doi.org/10.1253/circj.CJ-17-1352 